\title{
Security-enhanced Watermark Embedding and Transmission Based on Synchronized Chaotic Semiconductor Lasers
}

\section{Jun Wang}

Southwest University

\section{Xi Tang}

Southwest University

Fei Wang

Southwest University

Xiao-Dong Lin

Southwest University

Qiu-Lan Zheng

Southwest University

\section{Guang-Qiong Xia}

Southwest University

\section{Zheng-Mao Wu}

Southwest University

\section{Tao Deng ( $\sim$ dengt@swu.edu.cn )}

Southwest University https://orcid.org/0000-0001-5824-273X

\section{Research Article}

Keywords: Semiconductor laser, chaos synchronization, Arnold scrambling, watermarking encryption algorithm, chaotic secure communication

Posted Date: March 2nd, 2022

DOI: https://doi.org/10.21203/rs.3.rs-1375359/v1

License: (c) (i) This work is licensed under a Creative Commons Attribution 4.0 International License. Read Full License 


\title{
Security-enhanced watermark embedding and transmission based on synchronized chaotic semiconductor lasers
}

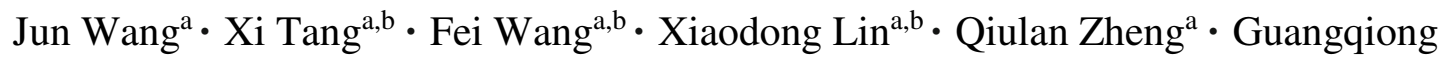 \\ $\mathrm{Xia}^{\mathrm{a}, \mathrm{b}} \cdot$ Zhengmao $\mathrm{Wu}^{\mathrm{a}, \mathrm{b}}$ and Tao Deng ${ }^{\mathrm{a}, \mathrm{b} *}$ \\ ${ }^{a}$ School of Physical Science and Technology, Southwest University, Chongqing \\ 400715, China \\ ${ }^{b}$ Chongqing key Laboratory of Micro\&Nano Structure Optoelectronics, Chongqing \\ 400715, China
}

*Corresponding author. E-mail: dengt@swu.edu.cn

\begin{abstract}
In this paper, we propose and numerically demonstrate a chaotic watermarking encryption and secure transmission scheme based on two synchronized response lasers (RLs) subject to identical injection from a driving laser (DL). In this proposed system, the chaos signals from DL can be optimized after passing through a phase modulator and a dispersion component, then the chaotic signal with large bandwidth and low TDS is obtained. Simultaneously injecting this chaotic signal into two identical RLs, high-quality chaos synchronization between two RLs can be realized. Both synchronized chaotic signals are respectively divided into two parts. One is used to generate shared keys required by the watermarking encryption, and the other is used as chaotic carrier to realize the secure message transmission. After introducing the synchronized optical chaos into the watermarking encryption algorithm, the normalized correlation of the extracted watermark without long-distance transmission is 0.9554 and can still maintain above 0.9 over $200 \mathrm{~km}$ transmission fiber. The message transmission and watermark extraction have good robustness to the fiber parameter mismatches, and excellent performances can be maintained even within $20 \%$ mismatches range. Moreover, the security of the watermarking encryption algorithm and the message transmission can be guaranteed simultaneously.
\end{abstract}

Keywords Semiconductor laser, chaos synchronization, Arnold scrambling, watermarking encryption algorithm, chaotic secure communication.

\section{Introduction}

With the development of communication technology, telemedicine has become a common means of remote diagnosis and treatment, which can further alleviate the structural imbalance of medical resources. During the whole telemedicine process, the medical staff can diagnose, evaluate and treat patients through the telemedicine platform. Meanwhile, medical images, patients' electronic medical records (EMR) and other private information need to be transmitted at both ends through the public 
network, which can bear huge risks tampering and stealing these privacy information.

As a security verification mechanism, watermarking technology is widely used in data transmission and distribution to provide additional protection in the existing message transmission process. Previous works about watermarking technology mainly focuses on embedding watermarking in the transform domain rather than in the time domain, because the small change of image pixel value will greatly affect the carrier image [1]. In the transform domain, the watermark image can be embedded into the frequency domain of the carrier image based on discrete cosine transform (DCT) [2], discrete Fourier transform (DFT) [3], or discrete wavelet transform (DWT) [4], which can make the image have enough robustness to attacks like rotation and cropping. And different transform technologies are combined to realize the imperceptibility of the carrier image [5-13].

The security of watermarking scheme is another important issue. During the watermarking encryption process, a chaos mapping is necessary to further improve the security of watermarking due to the ergodicity, sensitive dependence on initial conditions and parameters, and complex dynamic and deterministic behavior of chaos system. In 2018, Wang et al. proposed a memristive chaotic system and add it to the watermark embedding process by using DWT [14]. In the same year, Thakur et al. realized watermark embedding by non-sub-sampled contourlet transform (NSCT), redundant discrete wavelet transform (RDWT) and singular value decomposition (SVD), and 2-D logistic map based chaotic encryption is applied in carrier image [15]. In 2020, Alshoura et al. raised a new chaotic image watermarking scheme based on SVD and IWT with logistic and sine maps [16]. However, in these methods, the key parameters of the chaotic map need to be exchanged at both transmission terminals, which will undoubtedly increase the risks of the system. Moreover, compared with chaotic map, chaos generated by semiconductor laser has unique advantages including high complexity, large bandwidth and noise-like characteristics. Therefore, the application of chaotic system based on semiconductor laser in digital watermarking algorithm has become a challenging topic.

In this paper, the bandwidth and time delay signatures of the chaotic output from the driving laser (DL) with optical feedback can be optimized using a phase modulator (PM) and a dispersion component (De), and then the chaos signal is 
simultaneously injected into the response laser 1 (RL1) and response laser 2 (RL2). Under suitable injection parameters, two synchronized chaos outputs can be obtained, which are used to generate the key parameters of the watermarking algorithm and the chaos carrier of message transmission. Consequently, double-encryption chaotic watermarking embedding and transmission is realized. Moreover, the influence of transmission length and the mismatches of fiber parameters on the watermarking transmission and extraction was further investigated. Also, the security of the proposed watermarking embedding and transmission scheme is preliminarily discussed.

\section{Theoretical Model}

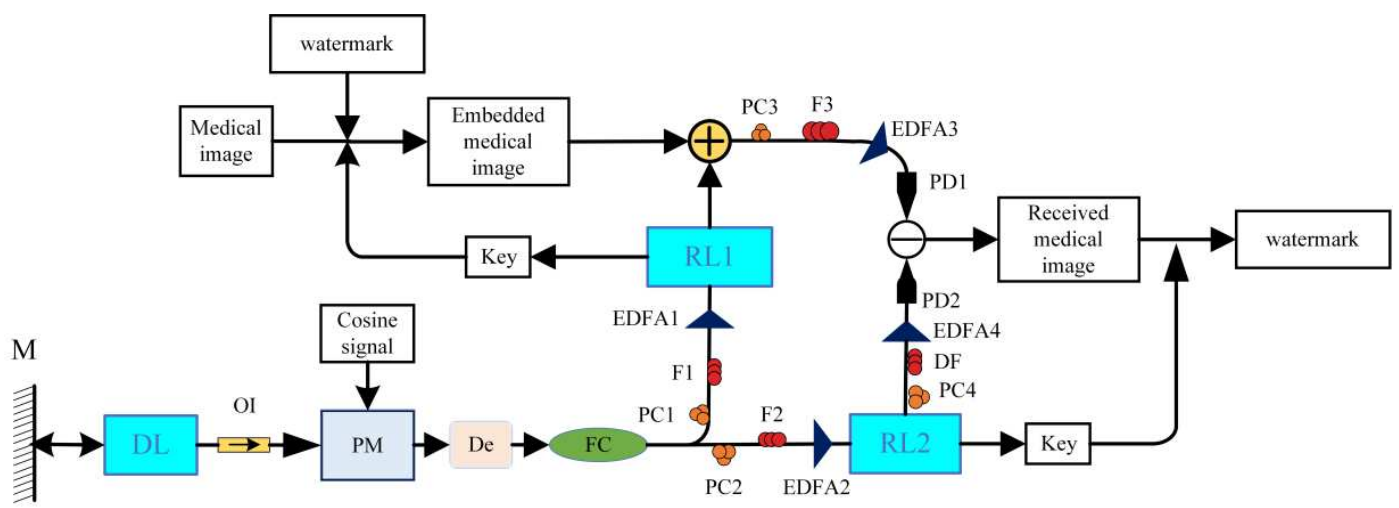

Fig. 1 Schematic diagram of watermark embedding and transmission system. M: mirror; DL: driving laser; PM: phase modulator; De: dispersion component; RL: response laser; F: fiber; FC:50/50 fiber coupler; DF: delay fiber; OC: optic circulator; PC: polarization controller; PD: photodetector; EDFA: erbium-doped optical fiber amplifier.

Fig. 1 shows the system configuration for security-enhanced watermark embedding and transmission based on two synchronized optical chaos from semiconductor laser. A driving laser (DL) is routed into chaos under suitable optical feedback. Then the chaotic signal output from the DL is divided into two beams after passing through a phase modulator (PM) and a dispersion component (De). One is injected into response laser 1 (RL1) through fiber link 1 (F1), and the other is injected into response laser 2 (RL2) through fiber link 2 (F2). Under appropriate parameters, RL1 and RL2 can achieve excellent chaotic signals with low TDS and broad bandwidth, and high-quality chaos synchronization can be realized [18]. Based on chaos synchronization between two RLs, security-enhanced watermark embedding and transmission can be achieved. In the process of watermark encryption, the 
synchronized chaos signals from two RLs are respectively used as key generators to control the watermark embedding process. During the message transmission, chaos masking (CM) [19] is used to hide image message with embedded watermark in the chaotic carrier of RL1, which is injected into a photodetector 1 (PD1) through fiber link F3. Meanwhile, one part of the chaos output of RL2, as a reference signal, is sent into PD2 for decoding message. Then, message can be recovered by comparing the difference between two outputs of PD1 and PD2 and filtering high-frequency components with a low pass filter. Finally, watermark can be extracted from the image through the adopted watermarking extraction method.

\subsection{Watermarking encryption and extraction}

In this section, the watermark embedding and extraction process will be introduced in detail. The original medical image (OMI, carrier image) is embedded with an electronic medical record (EMR, watermarking image) to produce the embedded image (EI). Here, the symbol image "T" is used to represent EMR, the size of OMI and EMR are respectively $256 * 256$ and $32 * 32$. The embedding process is shown below:

(a) The outputs of RLs are respectively sampled at equal step to obtain two sequences of the same length $M$. Then create two new binary sequences with M-1 length through comparing the value between two adjacent sampling points. If the sampling value is greater than that of the previous point, the point value in new sequence is set as 1 . Otherwise, it is 0 . Finally, convert these two binary sequences to decimal numbers between 0 and 255 .

(b) The Arnold scrambling function is used to scramble the pixel points of the carrier image, and then embed the watermark into the scrambled image, which can improve the security. The mathematical model of Arnold scramble is shown below:

$$
\left[\begin{array}{l}
x_{n+1} \\
y_{n+1}
\end{array}\right]=\left[\begin{array}{cc}
1 & b \\
a & a b+1
\end{array}\right]\left[\begin{array}{l}
x_{n} \\
y_{n}
\end{array}\right] \bmod (N)
$$

and the inverse Arnold scramble is shown:

$$
\left[\begin{array}{l}
x_{n+1} \\
y_{n+1}
\end{array}\right]=\left[\begin{array}{cc}
a b+1 & -b \\
-a & 1
\end{array}\right]\left[\begin{array}{l}
x_{n} \\
y_{n}
\end{array}\right] \bmod (N)
$$

where $x_{n}\left(y_{n}\right)$ and $x_{n+1}\left(y_{n+1}\right)$ respectively represents the positions of pixels in the gray image before and after transformation, $a$ and $b$ are the scrambling parameters, $n$ is the times of Arnold scrambling transformations, $N$ is the length 
or width of the image, mod represents modular operation. The parameters of $a, b$, $n$ are determined by the decimal number generated in step (a).

(c) By the discrete wavelet transforming (DWT), the scrambled medical image is decomposed into four parts: LL, LH, HL and HH. LL is a low-frequency subband, and $\mathrm{HL}, \mathrm{LH}, \mathrm{HH}$ are three high-frequency subbands. After performing discrete cosine transform (DCT), the four sidebands are respectively transformed into LL1, LH1, HL1 and HH1, and then divided into four $8 * 8$ blocks.

(d) Pick the center point of each $8 * 8$ block of LL1, LH1, HL1 and HH1, and replace the original value with the average of the four points around the selected point. Next, divide EMR into four pieces, and then select appropriate parameters to embed them into the $8 * 8$ block of LL1, LH1, HL1 and HH1, respectively.

(e) Perform inverse DCT (IDCT) and inverse DWT (IDWT) transformations on the DCT coefficients that have been embedded with EMR, and then an inverse Arnold scrambling are adopted to obtain the carrier image with embedded EMR image.

At the receiving end, an inverse process of watermark embedding is used to extract the complete watermark image.

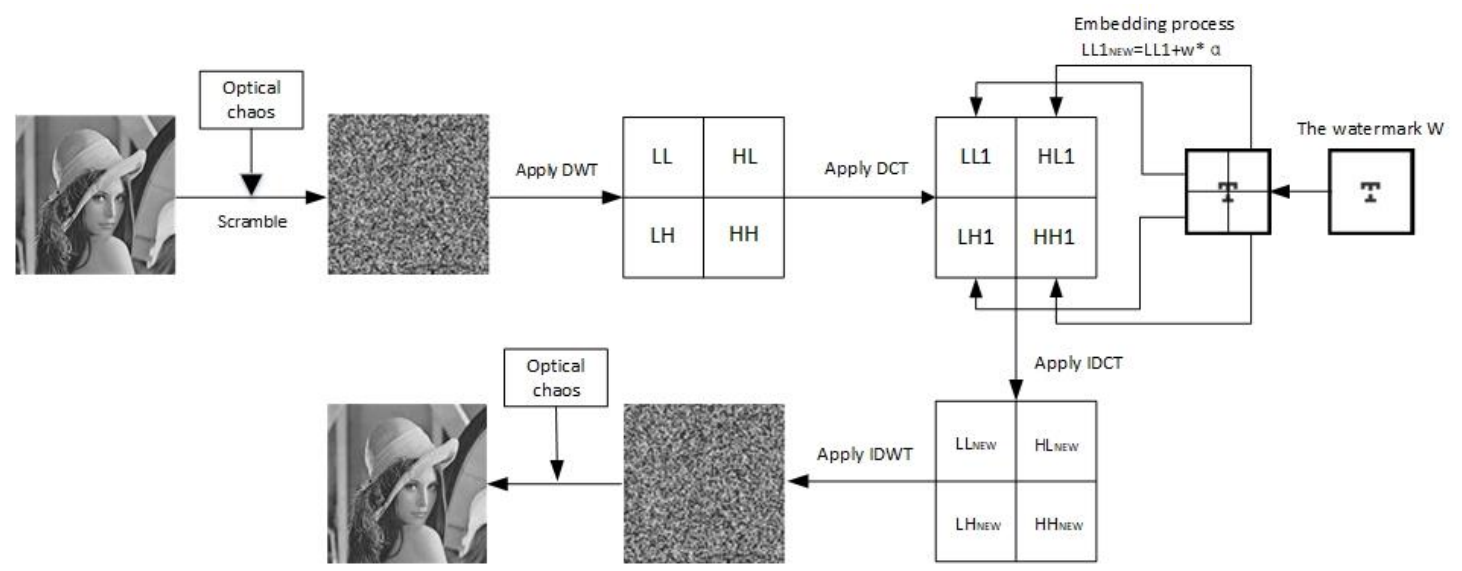

Fig. 2 The proposed watermark embedding process

\section{2 security-enhanced watermark transmission}

In this section, chaotic output from RL1 is used as carrier to hide the medical image with embedded watermark, which can be decrypted by the reference signals from RL2 in the receiving end.

The modified Lang-Kobayashi equation is used to investigate the dynamic characteristics of DL and RLs by considering the feedback term and the injection term, and can be expressed as [20]: 


$$
\begin{aligned}
& \frac{d E_{D L}}{d t}=\frac{1}{2}(1+i \alpha)\left(G_{D L}-\frac{1}{\tau_{p}}\right) E_{D L}+k_{d} E_{D L}\left(t-\tau_{\mathrm{d}}\right) \exp \left(-j \omega_{D L} \tau_{d}\right)+\sqrt{2 \beta N_{D L}} \xi_{D L}(t) \\
& \frac{d E_{R L 1, R L 2}}{d t}=\frac{1}{2}(1+i \alpha)\left(G_{R 1, R 2}-\frac{1}{\tau_{p}}\right) E_{R L 1, R L 2}+\sigma_{R L 1, R L 2} E_{\mathrm{inj}}\left(t-\tau_{i}\right)+\sqrt{2 \beta N_{R L 1, R L 2}} \xi_{R L 1, R L 2}(t) \\
& \frac{d N_{D L, R L 1, R L 2}}{d t}=\frac{I}{q}+\frac{N_{D L, R L 1, R L 2}(t)}{\tau_{n}}-G_{D L, R 1, R 2}\left|E_{D L, R 1, R 2}(t)\right|^{2} \\
& \mathrm{G}_{D L, R L 1, R L 2}(t)=\frac{g\left[N_{D L, R L 1, R L 2}-N_{0}\right]}{1+\varepsilon\left|E_{D L, R L 1, R L 2}(t)\right|^{2}}
\end{aligned}
$$

where the subscripts DL, RL1, and RL2 stand for driving laser, response laser 1, and response laser 2, respectively. $E$ is the complex amplitude of the optical field and $N$ represents the carrier number. $\alpha$ is the linewidth enhancement factor, $G_{D L, R L 1, R L 2}$ is the gain coefficient, $\tau_{p}$ is the photon lifetime, $k$ is the feedback parameter for DL, $\sigma_{R L 1, R L 2}$ is the injection coefficient, $\tau_{d}$ is the feedback delay, $\omega$ is the angular frequency, $q$ is the electronic charge, $I$ is the bias current, $g$ is the differential gain coefficient, $\varepsilon$ is the gain saturation factor, $N_{0}$ is the transparent carrier number, $\beta$ is the spontaneous emission rate, $\xi$ presents the Gaussian white noise.

The phase modulation is implemented by a typical $\mathrm{LiNiO}_{3}$ phase modulator, which is mathematically described as:

$$
E_{\text {out }}=E_{\text {in }} \exp \left(i \pi \frac{V_{\text {key }}(t)}{V_{\pi}}\right)
$$

where the $V_{\pi}$ is the half-wave voltage of PM, the driving signal $V_{k e y}$ is mathematically described as $V_{\text {Key }}(t)=A_{0} \cos \left(2 \pi f_{0} t\right)$, where $A_{0}$ stands for the amplitude and $f_{0}$ is the frequency. The dispersion component De is a dispersion media such as dispersive fiber and the corresponding transfer function in the frequency domain can be described as [21]:

$$
H_{D e}=\exp \left(i \frac{1}{2} \beta_{2} L \omega^{2}\right)
$$

where $L$ is the length of the dispersive fiber, $\beta_{2}=-D e \lambda^{2} / 2 \pi c$ is the group velocity dispersion, $D e$ is the dispersion coefficient, $\lambda$ is the wavelength of the chaotic carrier and $c$ is the velocity of light in vacuum. Applying the inverse Fourier transformation to Eq. (6), we can obtain the pulse response in the time domain:

$$
h_{D e}=\exp \left(-i \frac{\omega_{0}}{2 \lambda_{0} D e L} t^{2}\right)
$$


The signal propagation in optical fiber channels could be described by the following nonlinear Schrödinger equation [22]:

$$
i \frac{\partial E(z, T)}{\partial z}=-\frac{i}{2} \alpha E+\frac{1}{2} \beta_{2} \frac{\partial^{2} E}{\partial T^{2}}-\gamma|E|^{2} E
$$

where $E(\mathrm{z}, \mathrm{T})$ presents the slowly varying amplitude of the optical field, $z$ is the propagation length, $\alpha$ is the fiber loss constant, $\beta_{2}$ is the second-order dispersion coefficient, $\gamma$ is the nonlinear Kerr factor.

The bandwidth and TDS characteristics of the DL and RLs outputs are respectively evaluated by calculating the effective bandwidth (EBW) and auto-correlation function (ACF). The efficient bandwidth is defined as the span between the DC and the frequency where $80 \%$ energy is contained in the RF spectrum [23]. The ACF is defined as [24]:

$$
\mathrm{C}_{i, j}(\Delta t)=\frac{\left\langle\left[I_{\mathrm{i}}(t)-\left\langle I_{i}(t)\right\rangle\right] *\left[I_{j}(t-\Delta t)-\left\langle I_{j}(t-\Delta t)\right\rangle\right]\right\rangle}{\sqrt{\left\langle\left[I_{i}(t)-\left\langle I_{i}(t)\right\rangle\right]^{2}\right\rangle *\left\langle\left[I_{j}(t-\Delta t)-\left\langle I_{j}(t-\Delta t)\right\rangle\right]^{2}\right\rangle}}
$$

where $I(t)=|E(t)|^{2}$ denotes the intensity, $\Delta t$ denotes the time shift and $\langle\cdot\rangle$ stands for the time averaging. When $i$ and $j$ are identical, the peak value $\sigma$ locating at the feedback delay time in ACF is used to evaluate the matching degree between the time series and its time-shifted replica. When $i$ and $j$ represent different lasers, $C$ is corresponding to the cross-correlation function (CCF) and represents the synchronization quality between two chaotic signals.

\section{Results and discussion}

During the simulations, the fourth-order Runge-Kutta algorithm is adopted to solve the rate equations, and the split-step Fourier method is used to solve the nonlinear Schrödinger equation. The typical intrinsic parameters of the lasers are set as [17]: $\lambda=1550 \mathrm{~nm}, \quad \alpha=5, \quad g=1.5 \times 10^{-8} p s^{-1}, \quad \varepsilon=1 \times 10^{-7}, \quad \beta=1.5 \times$ $10^{-6} \mathrm{~ns}^{-1}, \tau_{p}=2 \mathrm{ps}, \tau_{e}=2 \mathrm{~ns}, N_{0}=1.5 \times 10^{8}, I=2 I_{t h}, I_{t h}=14.7 \mathrm{~mA}$, $k_{d}=15 n s^{-1}, \tau_{d}=3 n s$. 


\subsection{Generation of chaos signals with broad bandwidth and low TDS}
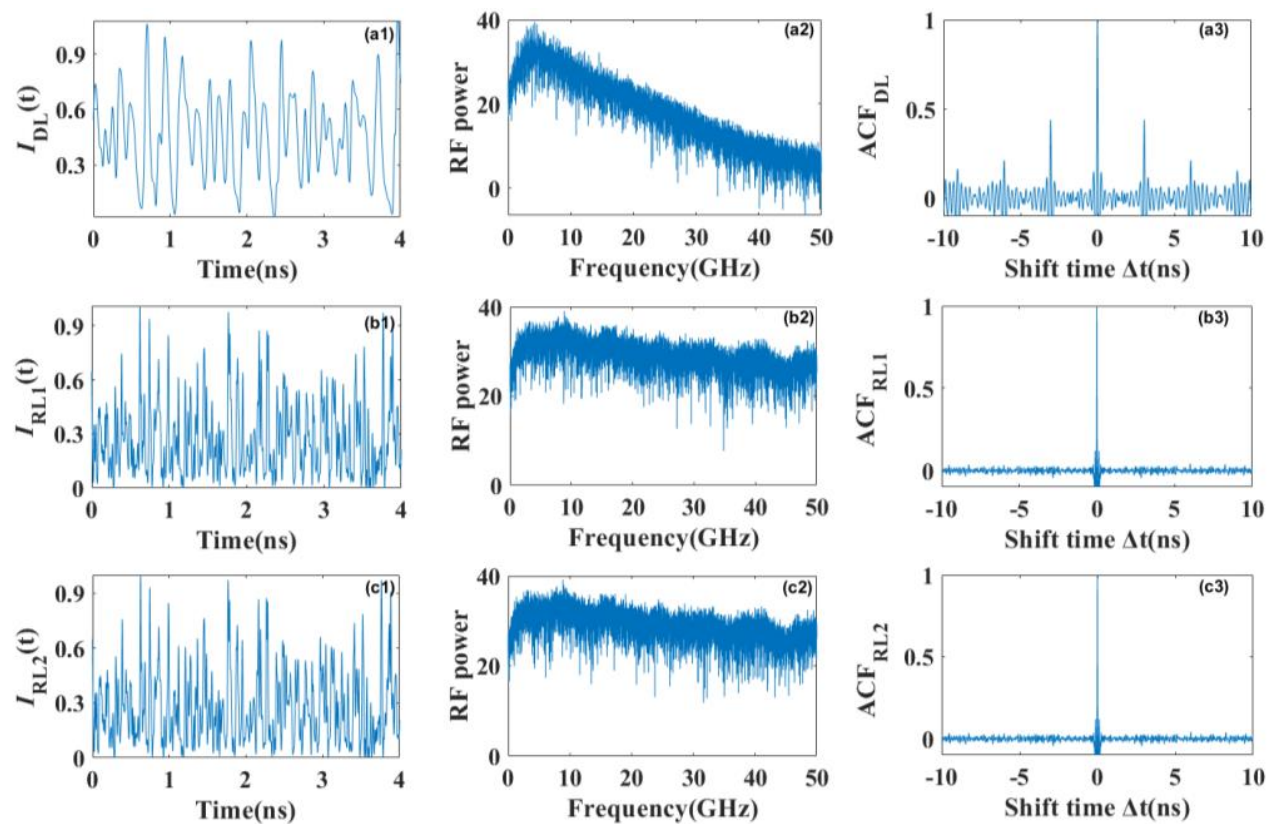

Fig. 3 Temporal waveform (first column), RF spectrum (second column), and ACF (third column) of the DL (first row) and RLs (second and third row).

Fig. 3 shows the time series, RF spectra, and ACF curves of the DL and the RLs. Compared with $12.4 \mathrm{GHz}$ chaos signal of DL, the effective bandwidth of RLs outputs are extended to about $39.6 \mathrm{GHz}$. Moreover, there is no subsidiary peak in the ACF curves and TDS of chaotic signal is suppressed to a very low level, which indicates that the complexity of chaos signals is greatly improved. The performance optimization of original chaos signals mainly originates from the combination of the spectrum expansion effect of PM and the phase-to-intensity conversion of dispersion component [17], which can provide an excellent chaos resource for future security-enhanced high-speed chaos communication.

\section{2 chaos synchronization}

In order to investigate the synchronization properties between arbitrary two chaotic signals from DL and two RLs, Fig. 4 displays the cross-correlation coefficients between arbitrary two chaotic signals when the system configuration is symmetric and the length of fiber link $F_{1}$ (or $F_{2}$ ) is $25 \mathrm{Km}$. During the simulations, the parameters of $F_{1}\left(\right.$ or $\left.F_{2}\right)$ are chosen as [25]: $\alpha=0.2 \mathrm{~dB} / \mathrm{km}, \quad \beta_{2}=-0.1 \mathrm{ps}^{2} / \mathrm{km}$, $\gamma=1.5 \mathrm{~W}^{-1} / \mathrm{km}$. When the injection strength is $60 \mathrm{~ns}^{-1}$, zeros-lag high-quality chaos synchronization between two RLs is achieved and the maximal $C_{R L 1, R L 2}$ is almost 1 at $\Delta t=0$ while that between DL and arbitrary RLs is only about 0.1 , as shown in 
Fig. 4(a1) -(a3).
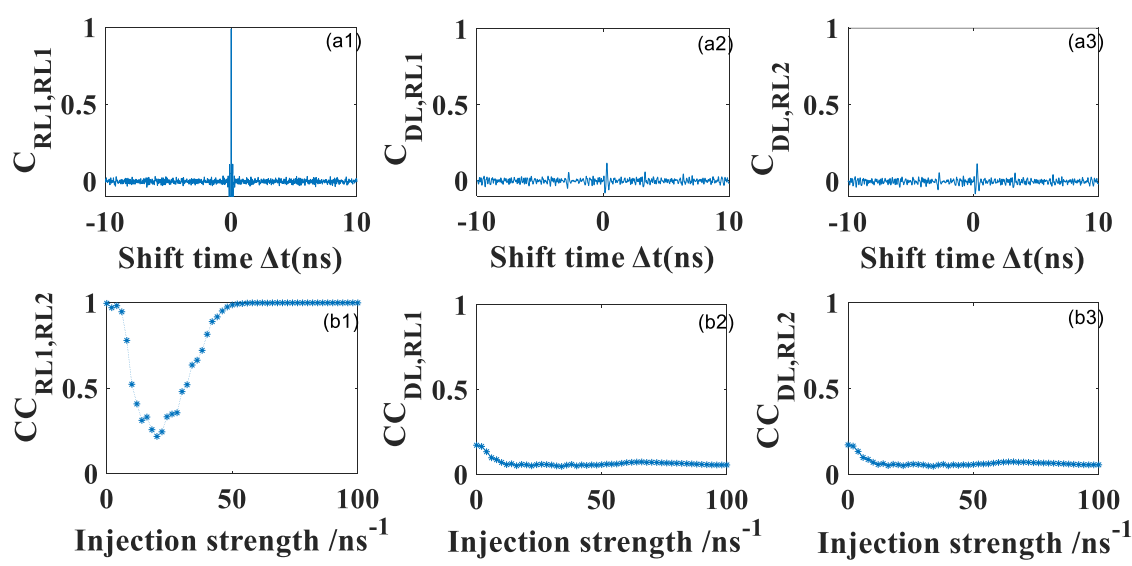

Fig. 4 The cross-correlations between arbitrary two chaos outputs from DL and two RLs.

Fig. 4(b1) -(b3) further show the variation of the correlation coefficients with the injection strength from DL to two RLs. From these diagrams, one can see that, for relatively small injection strength, the same seed induces two RLs to output almost identical chaos signals due to nonlinear effect in lasers and high-quality chaos synchronization between two RLs can be realized. Then the synchronization quality can be deteriorated due to complex dynamical outputs with increasing the injection strength. Once the injection strength exceeds a certain level, high-quality chaos synchronization is again achieved due to the injection locking. For all simulated injection strength, the synchronization coefficient between DL and arbitrary RL is very low. Obviously, these results can guarantee the security of information transmission between two RLs at a certain extent.

\subsection{Watermark embedding, transmission and extraction}

Based on high-quality chaos synchronization of two RLs, the message encryption is performed by adopting chaos modulation method, which can be mathematically described as $\left|E_{m m}(t)\right|=\left|E_{R L 1}(t)\right|\left[1+M_{m} m(t)\right]$, where $E_{m m}(t)$ denotes the modulated carrier, $m(t)$ is the original message, $M_{m}$ is the modulation depth and 0.05 is selected to guarantee that the message is well hidden in the chaotic carrier [26,27]. The message is recovered by the direct subtraction decoding which is described as $m^{\prime}(t)=\operatorname{LPF}\left[\left|E_{m m}(t)\right|^{2}-\left|E_{R L 2}(t)\right|^{2}\right]$, where LPF means the recovered message is filtered by a low-pass five-order Butterworth filter. The communication performance is quantified by calculating the Q-factor of recovery message, which is defined as: 


$$
Q=\frac{\left\langle I_{1}\right\rangle-\left\langle I_{0}\right\rangle}{\sigma_{1}+\sigma_{0}}
$$

where $\left\langle I_{1}\right\rangle$ and $\left\langle I_{0}\right\rangle$ stand for the average power of bits " 1 " and " 0 ", respectively. $\sigma_{1}$ and $\sigma_{0}$ are the corresponding standard deviations. A Q-factor value greater than 6 corresponds to a satisfactory BER about $10^{-9}$ [25].

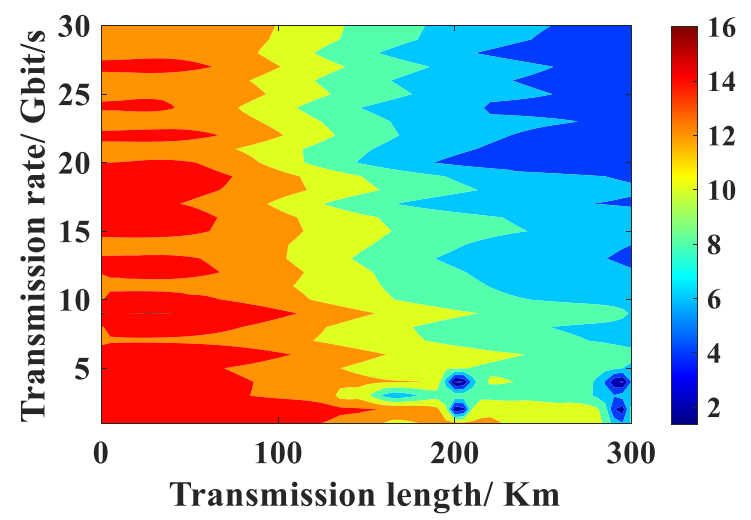

Fig. $5 \mathrm{Q}$ factor of the message decryption in the proposed chaotic communication system.

In order to evaluate the communication performance under different transmission distances, pseudorandom message is transmitted and Q-factor evolution for recovered message as a function of the fiber channel length and transmission rate is shown in Fig. 5. From this diagram, one can see that, for a certain transmission rate, the Q-factor of the decoded message shows that as the fiber length increases, the transmission quality remains high for a short time and then gradually decreases at the critical transmission length. It is worth noting that with the increase of the transmission rate, for a certain transmission length, the Q-factor also shows a decrease trend on the whole, and the critical transmission length corresponding to the beginning of the decrease of the Q-factor also gradually decreases.

To explore the watermark embedding, transmission and extraction performances, we use a sample "T" as watermark image to represent electronic medical record (EMR), which is embedded into an original medical image and transmitted into terminal through the proposed chaos secure communication system, then the embedded watermark can be extracted. Here, $10 \mathrm{Gbit} / \mathrm{s}$ image messages are transmitted. Normalized correlation coefficient (NC) and peak signal-to-noise ratio (PSNR) are used to evaluate the quality of EMR and carrier image respectively, which are defined as: 


$$
\begin{gathered}
N C\left(I_{1}, I_{2}\right)=\frac{\sum_{i=1}^{M} \sum_{j=1}^{N}\left[I_{1}(i, j)-\mu_{1}\right]\left[I_{2}(i, j)-\mu_{2}\right]}{\sqrt{\sum_{i=1}^{M} \sum_{j=1}^{N}\left[I_{1}(i, j)-\mu_{1}\right]^{2}} \sqrt{\sum_{i=1}^{M} \sum_{j=1}^{N}\left[I_{2}(i, j)-\mu_{2}\right]^{2}}} \\
P S N R=10 \log \frac{M * N^{*} 255^{2}}{\sum_{i, j}\left[I_{1}(i, j)-I_{2}(i, j)\right]^{2}}
\end{gathered}
$$

where $\mu_{1}$ and $\mu_{2}$ denote the mean values of $I_{1}$ and $I_{2}$, respectively. When the original and extracted watermark image closely resembles one another, $\mathrm{NC} \approx 1$. In addition, when PSNR is over $30 \mathrm{~dB}$, it is difficult to distinguish the carrier image with and without embedded watermark.

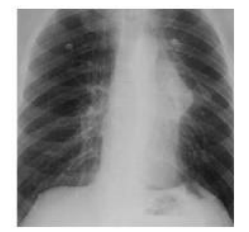

(a)

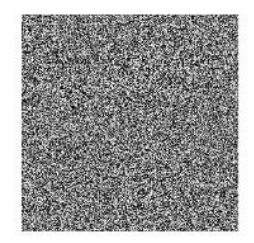

(d)

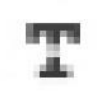

(b)

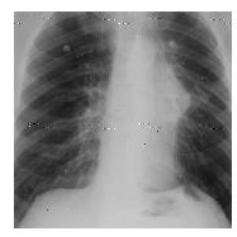

(e)

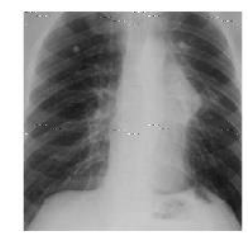

(c)

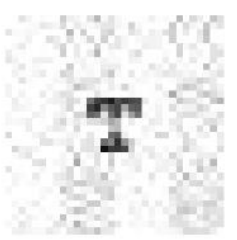

$(f)$

Fig. 6 The watermark embedding, transmission and extraction when the transmission length is $150 \mathrm{~km}$. (a) original medical image, (b) original EMR, (c) medical image with embedded watermark, (d) chaos masked medical image with embedded watermark (e) decoded medical image with embedded watermark after transmission. $(f)$ extracted EMR.

Fig. 6 presents the results of watermark embedding, secure transmission and watermark extraction in detail after transmitting over $150 \mathrm{~km}$ fiber channel. From these diagrams, one can see that the watermark can be embedded in the original medical image and hidden based on chaotic encryption method, as shown in Fig. 6(c) and (d). Then, the carrier image can be successfully decrypted after transmission over $150 \mathrm{~km}$ fiber channel, as shown in Fig.6 (e), and PSNR can be maintained over 30dB, which means that almost no information lost during the transmission. Moreover, Fig. 6 (f) shows the extracted watermark, and NC between original and extracted watermark is 0.9554, which denotes the successful watermark embedding and extraction based on the synchronized chaos system. 

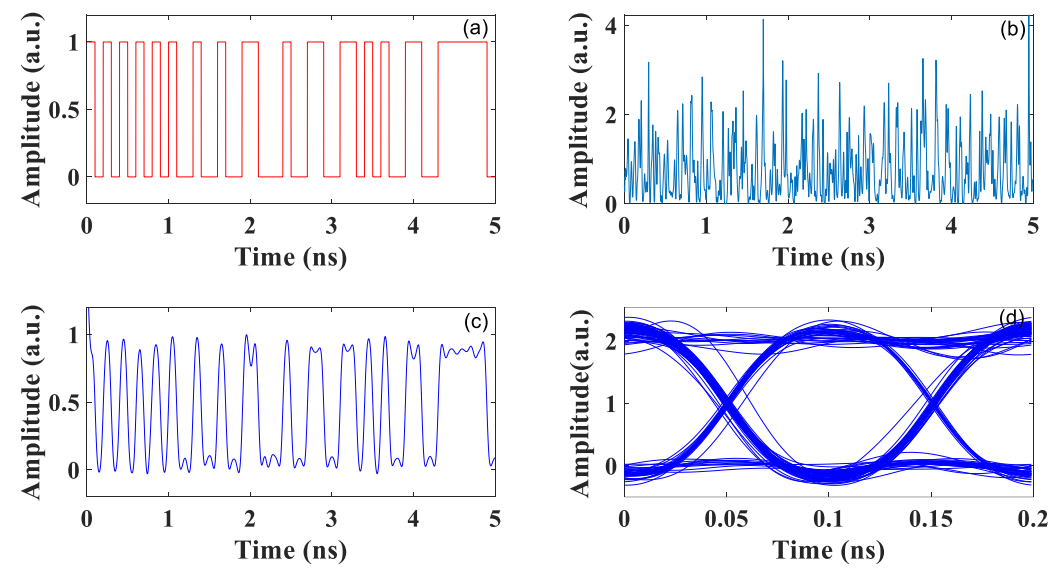

Fig. 7 Transmission results after propagating $150 \mathrm{~km}$ in the SMF channel. (a) Original message; (b) Chaotic carrier with message; (c) Decoded message; (d) Eye diagram of decoded message.

For investigating the secure message transmission performance in detail, Fig. 7 shows the transmission results of encoded image for the case in Fig. 6, here only part of encoded messages are displayed. From these diagrams, one can see that the encoded messages can effectively hidden in chaotic carrier, as shown in Fig. 7(b). After transmitting over $150 \mathrm{~km}$ fiber, the messages can be successfully recovered with some distortions and the corresponding eye diagram is wide-open and clear, as shown in Fig. 7(c) and (d), which means the Q-factor of decoded messages is higher than the conventional communication requirement with $\mathrm{Q}$-factor $\geqslant 6[28,29]$. These results means that the proposed system can realize secure message transmission.
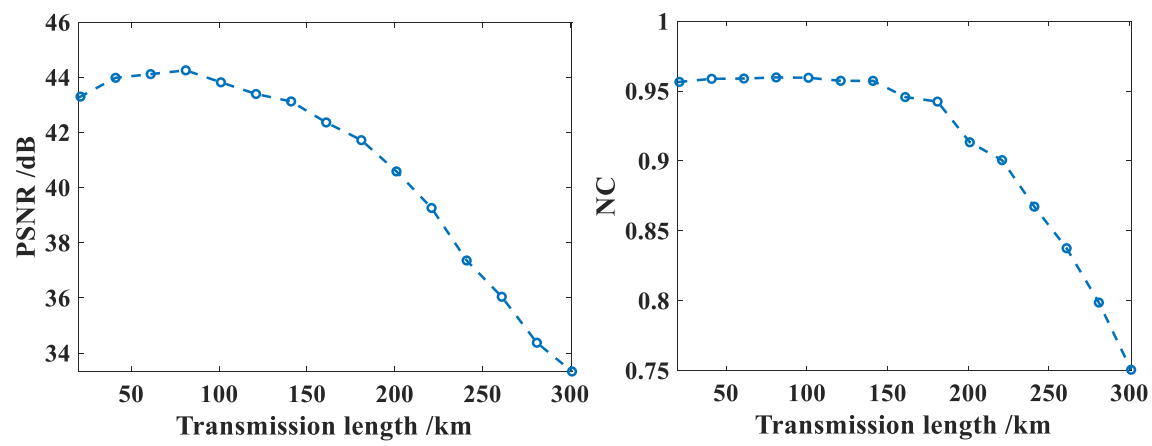

Fig. 8 The change of the PSNR value of medical images with embedded EMR and the NC value of EMR verse transmission length.

Fig. 8 further shows the variations of the PSNR of medical images and NC value of EMR under different transmission lengths. From these diagrams, one can see that PSNR is almost over $40 \mathrm{~dB}$ after transmission over $200 \mathrm{~km}$ fiber channel, then gradually decreases, but maintains over $30 \mathrm{~dB}$ even for $300 \mathrm{~km}$ transmission length. Similarly, NC value of watermark first maintain at a relative high level and then 
gradually decrease with increasing the transmission length. These phenomena can be explained as that, the dispersion, nonlinear effects and noise in the long-distance fiber will distort the transmitted information, then the chaotic carrier cannot be completely removed in the decryption process. Therefore, with the increase of transmission length, the degree of signal distortion gradually increases. After a long distance transmission, although some information can be still obtained from the image, effective EMR images cannot be successfully extracted from the carrier image.

\subsection{Parameter mismatch robustness of the transmitted images}

It is well known that, in chaos synchronization system, the decoding quality is partly determined by the residual chaotic carrier, which also affects the performance of extracted watermark image. Normally, as for messages transmission and decoding in this proposed system, if $F_{3}$ owns the same characteristics as that of $D F$, the chaotic carrier can be completely eliminated during the process of message decoding. However, the parameters mismatch between $F_{3}$ and $D F$ may exist in reality. Thus, it is necessary to analyze the impact of mismatched fiber parameters including chromatic dispersion, power attenuation, fiber nonlinearities, and fiber length. Here, for simplification, we fix the parameters of $F_{3}$ and only respectively adjust one of these typical parameters such as $\beta_{2}, \alpha, \gamma$ and the length of $D F$. The relative parameter mismatches could be defined similar as
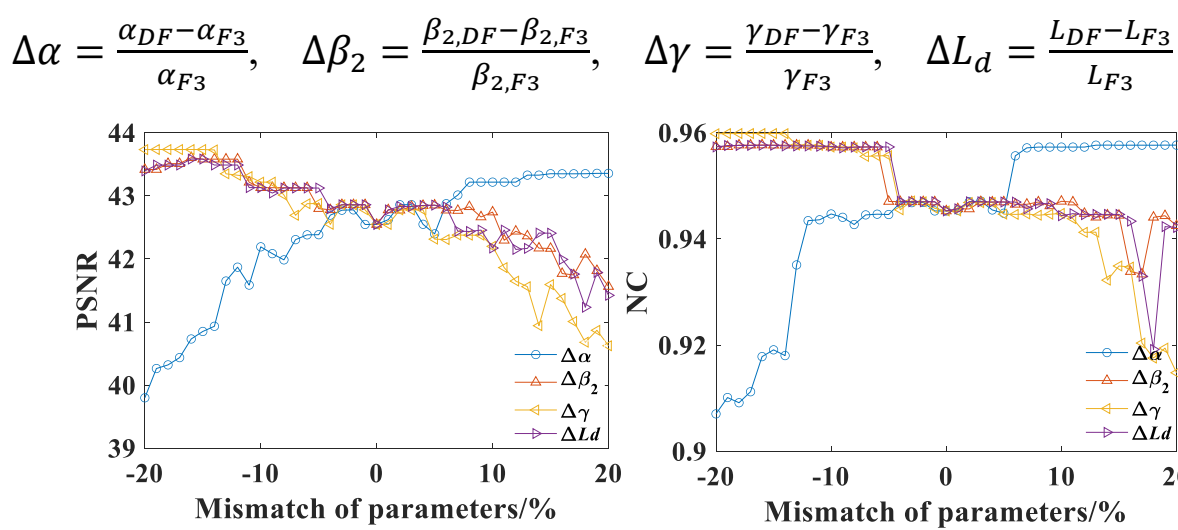

Fig. 9 PSNR and NC value as a function of the power attenuation, chromatic dispersion, nonlinear Kerr factor and length mismatch.

Fig. 9 displays the NC and PSNR evolutions for $10 \mathrm{~Gb} / \mathrm{s}$ message as a function of $\Delta \alpha, \Delta \beta_{2}, \Delta \gamma$ and $\Delta L_{d}$. One can observe that the PSNR and NC have similar evolution trend and relatively good robustness to the fiber parameter mismatches with $\pm 20 \%$. Compared with other parameters, the negative $\Delta \alpha$ mismatch has greater 
effect on PSNR and NC. It should noted that multiple parameter mismatches simultaneously exist in real scene, which can induce more complex evolution.

\subsection{Security evaluation}

\subsubsection{Security analysis of watermark embedding and extraction}

\section{A. Key space}

When the key space is larger than $2^{100}$, the proposed watermark embedding scheme is enough secure to resist the brute force attack [30,31]. The total key space includes the embedding position and the used chaotic sequence. Especially, the chaotic sequence generated from the disturbed semiconductor laser has highly randomness and is used to control the Arnold scrambling and hide the carrier image with embedded watermark, which can greatly extend the key space. Therefore, the system security can be effectively guaranteed even for some brute force attacks.

\section{B. Key sensitivity}

In order to evaluate the key sensitivity in our watermark embedding and extracting scheme. Two binary sequence $X_{1}$ and $X_{2}$ are respectively used as secret key at the receiving end. These two sequences are the same and all generated from step (a) of watermark embedding process, but the value of first point in $\mathrm{X}_{2}$ is artificially changed from 1 to 0 . Fig. 10 shows the watermark extraction results for these two cases. From these diagrams, one can see that, the watermark image can be successfully extracted with the key $X_{1}$, while the extraction is failure with $X_{2}$, which means that the watermark embedding and extracting scheme is sensitive to the secret keys and a tiny change can induce the failed watermark extraction. Therefore, synchronized chaos sequence generation from two RLs is a key issue in our proposed system.

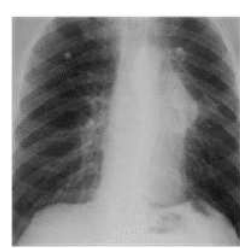

(a)

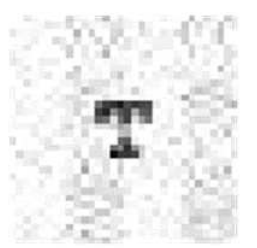

(b)

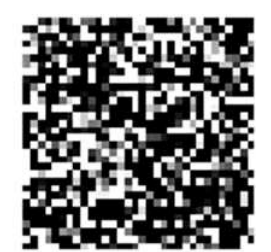

(c)

Fig. 10 The result of key sensitivity. (a) Original image with watermark. (b) Extracted watermark with $\mathrm{X}_{1}$. (c) Extracted watermark with $\mathrm{X}_{2}$. 


\subsubsection{Security analysis of watermark transmission}

Secure communication based on chaotic carrier generated by semiconductor laser has attracted wide attention due to its high security in hardware encryption. The security mainly depends on the difficulty of transmitter parameter identification and the sensitivity of synchronization quality to parameter mismatches. That is, the whole set of structural parameters and operational parameters play a key role during the communication between the transmitter and receiver. Usually, different attract scenarios can exist in the real communication [32,33]. In order to further embody the security of chaotic secure communication. Here, we take the most serious attract situation, in which the eavesdropper has stolen the structural parameters of RLs. Then the eavesdropper amplified the stolen transmitter signal from public channel and injected it into another illegal SL to achieve chaos synchronization for the illegal message extraction. Fig. 11 shows the decryption result under this type of illegal attack scenario. Apparently, eavesdropper cannot steal any effective information about no matter the carrier image or watermark image.

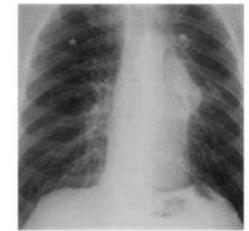

(a)

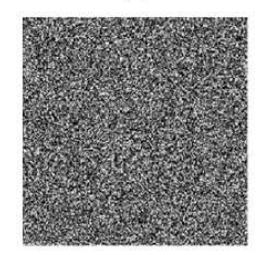

(c)

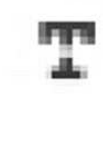

(b)

(d)

Fig. 11 illegal decryption result of synchronization utilization attack. (a) original carrier image. (b) original EMR. (c) stolen carrier image. (d) stolen EMR.

In this proposed system, two synchronized chaos signals are used for the watermark embedding, secure transmission and extraction. Above-mentioned security evaluations have demonstrated that, the double encryption method can effectively guarantee the security of privacy information of patients during the diagnosis and treatment process.

\section{Conclusion}

Based on a chaotic synchronization system consisted of two RLs subject to 
identical chaos optical injection, we propose and numerically investigate a chaotic watermarking encryption and transmission system. The simulated results show that, the chaotic signal with large bandwidth and low TDS is obtained after using a phase modulator and a dispersion component, and high-quality chaos synchronization is achieved between the two response lasers. The chaotic signals of RLs are divided into two parts, one part generates the shared keys of Arnold scrambling in the watermarking algorithm, and the other is used as the carrier of image transmission for chaotic secure communication. The EMR can be successfully embedded in OMI and securely transmitted over $200 \mathrm{~km}$ fiber channel, and the PSNR of carrier image is over $40 \mathrm{~dB}$ and the NC of EMR is maintained above 0.9 at the receiver end. Furthermore, the security of the watermarking embedding and transmission has also been discussed. The proposed double encryption method of watermark can greatly guarantee the privacy of patient and provide a new information management pattern for the future intelligent medical system.

Acknowledgements This work was supported by the National Natural Science Foundation of China (Grant No. 61875167), the Natural Science Foundation of Chongqing City (Grant cstc2019jcyj-msxmX0136), the Science and Technology Research Program of Chongqing Municipal Education Commission (Grant KJZD-K202100204).

\section{Compliance with ethical standards}

Conflict of interest The authors declare that they have no conflict of interest.

\section{Data availability}

All data generated or analyzed during this study are included in this published article.

\section{References}

1. Hsu, L. Y., Hu, H. T.: A reinforced blind color image watermarking scheme based on schur decomposition. IEEE Access 99: 107438-107452 (2019).

2. Patra, J. C., Phua, J. E., Bornand, C.: A novel DCT domain CRT-based watermarking scheme for image authentication surviving JPEG compression. Digit. Signal Prog. 20(6): 1597-1611 (2010).

3. Premaratne, P., Ko, C. C.: A novel watermark embedding and detection scheme for images in DFT domain. IET Conf. Proc. 2: 780-783 (1999).

4. Najafi, E.: A robust embedding and blind extraction of image watermarking based on discrete wavelet transform. Math. Sci. 11(4): 307-318 (2017).

5. Abdulrahman, A. K., Ozturk, S.: A novel hybrid DCT and DWT based robust watermarking 
algorithm for color images. Multimed Tools Appl. 78: 17027-17049 (2019).

6. Ali, M., Ahn, C. W., Pant, M., Siarrt, P.: An image watermarking scheme in wavelet domain with optimized compensation of singular value decomposition via artificial bee colony. Inf. Sci. 301: 44-60 (2015).

7. Makbol, N. M., Khoo, B. E., Rassem, T. H.: Block-based discrete wavelet transform-singular value decomposition image watermarking scheme using human visual system characteristics. IET Image Process 10(1): 34-52 (2016).

8. Roy, S., Pal, A. K.: A robust blind hybrid image watermarking scheme in RDWT-DCT domain using Arnold scrambling. Multimed Tools Appl. 76: 1-40 (2017).

9. Ali, M., Ahn, C. W., Pant, M.: A robust image watermarking technique using SVD and differential evolution in DCT domain. Optik 125(1):428-434 (2014).

10. Ling, H. C., Phan, C. W., Heng, S. H.: Advances in Image and Video Technology. In: Ho YS. (eds) PSIVT 2011. Lecture Notes in Computer Science, vol. 7087. pp. 257-266. Springer, Berlin, Heidelberg. (2011).

11. Singh, D., Singh, S. K.: DWT-SVD and DCT based robust and blind watermarking scheme for copyright protection. Multimed Tools Appl. 76(11): 13001-13024 (2017).

12. Singh, A. K.: Improved hybrid algorithm for robust and imperceptible multiple watermarking using digital images. Multimed Tools Appl. 76(6): 8881-8900 (2017).

13. Roy, S., Pal, A. K.: A robust blind hybrid image watermarking scheme in RDWT-DCT domain using Arnold scrambling. Multimed Tools Appl. 76(3): 3577-3616 (2017).

14. Wang, B., Zou, F. C., Zhang, Y.: New memritive chaotic system and the application in digital watermark. Optik 172: 873-878 (2018).

15. Thakur, S., Singh, A. K., Ghrera, S. P., Mohan, A.: Chaotic based secure watermarking approach for medical images. Multimed Tools Appl. 79(7-8), 4263-4276 (2020).

16. Alshoura, W. H., Zainol, Z., Teh, J. S., Alawida, M.: A new chaotic image watermarking scheme based on SVD and IWT. IEEE Access 8: 43391-43406 (2020).

17. Jiang, N., Zhao, A. K., Wang, Y. J., Liu, S. Q., Tang, J. M., Qiu, K.: Security-enhanced chaotic communications with optical temporal encryption based on phase modulation and phase-to-intensity conversion. OSA Contin. 2(12): 3422-3437 (2019).

18. Wu, J. G., Wu, Z. M., Xia, G. Q., Deng, T., Lin, X. D., Tang, X., Feng, G. Y.: Isochronous synchronization between chaotic semiconductor lasers over 40-km fiber links. IEEE Photonics Technol. Lett. 23(24): 1854-1856 (2011).

19. Jiang, N., Xue, C., Lv, Y., Qiu, K.: Physically enhanced secure wavelength division multiplexing chaos communication using multimode semiconductor lasers. Nonlinear Dyn. 86(3), 1937-1949 (2016).

20. Lang, R., Kobayashi, K.: External optical feedback effects on semiconductor injection laser properties. IEEE J. Quantum Electron. 16(3):347-355 (1980).

21. Cheng, M., Deng, L., Li, H., Liu, D. M.: Enhanced secure strategy for electro-optic chaotic systems with delayed dynamics by using fractional Fourier transformation. Opt. Express 22(5): 5241-5351 (2014)

22. Li, N., Wu, Z., Lin, X., Tang, X., Xia, G. Q., Deng, T.: Bidirectional digital image secure transmission and recognition based on a long-distance chaos synchronization system with machine learning capability. Nonlinear Dyn. 104, 2745-2758 (2021)..

23. Wang, A. B., Wang, Y. C., Yang, Y. B., Zhang, M. J., Xu, H., Wang, B. J.: Generation of 
flat-spectrum wideband chaos by fiber ring resonator. Appl. Phys. Lett. 102(3): 343 (2013).

24. Wang, A. B., Yang, Y. B., Wang, B. J., Zhang, B. B., Li, L., Wang, Y. C.: Generation of wideband chaos with suppressed time-delay signature by delayed self-interference. Opt. Express 21(7): 8701-8710 (2013).

25. Bogris, A., Kanakidis, D., Argyris, A., Syvridis, D.: Performance characterization of a closed-loop chaotic communication system including fiber transmission in dispersion shifted fibers. IEEE J. Quantum Electron. 40(9): 1326-1336 (2004).

26. Jiang, N., Pan, W., Luo, B., Yan, L. S., Xiang, S. Y.: Simultaneous unidirectional and bidirectional chaos-based optical communication using hybrid coupling semiconductor lasers. Sci. China Inf. Sci. 57: 1-11 (2014).

27. Li, N. Q., Susanto, H., Cemlyn, B., Henning, I. D., Adams, M. J.: Secure communication systems based on chaos in optically pumped spin-VCSELs. Opt. Lett. 42: 3494-3497 (2017).

28. Xie, Y. Y., Nikdast, M., Xu, J., Zhang, W., Li, Q.: Crosstalk noise and bit error rate analysis for optical network-on-chip. Design Automation Conference 657-660 (2010).

29. Shake, I., Takara, H., Uchiyama, K., Yamabayashi, Y.: Quality monitoring of optical signals influenced by chromatic dispersion in a transmission fiber using averaged Q-factor evaluation. IEEE Photonics Technol. Lett. 13(4):385-387 (2001).

30. Wu, X. L., Zhu, B., Hu, Y. T., Ran, Y. M.: A novel color image encryption scheme using rectangular transform-enhanced chaotic tent maps. IEEE Access 5: 6429-6436 (2017).

31. Seyedzadeh, S. M., Mirzakuchaki, S.: A fast color image encryption algorithm based on coupled two-dimensional piecewise chaotic map. Signal Process. 92(5): 1202-1215 (2012).

32. Jiang, N., Zhang, C., Qiu, K.: Secure passive optical network based on chaos synchronization. Opt. Lett. 37(21): 4501-4503 (2012).

33. Bogris, A., Argyris, A., Syvridis, D.: Encryption efficiency analysis of chaotic communication systems based on photonic integrated chaotic circuits. IEEE J. Quantum Electron. 46(10): 14211429 (2010). 